\title{
Sensors Based on Thin-Film Coated Cladding Removed Multimode Optical Fiber and Single-Mode Multimode Single-Mode Fiber: A Comparative Study
}

\author{
Ignacio Del Villar, Abian B. Socorro, Miguel Hernaez, Jesús M. Corres, \\ Carlos R. Zamarreño, Pedro Sanchez, Francisco J. Arregui, and Ignacio R. Matias
}

Electrical and Electronic Engineering Department, Public University of Navarra, 31006 Pamplona, Spain

Correspondence should be addressed to Ignacio Del Villar; ignacio.delvillar@unavarra.es

Received 31 March 2015; Accepted 4 June 2015

Academic Editor: Hai-Feng Ji

Copyright ( 92015 Ignacio Del Villar et al. This is an open access article distributed under the Creative Commons Attribution License, which permits unrestricted use, distribution, and reproduction in any medium, provided the original work is properly cited.

Two simple optical fibre structures that do not require the inscription of a grating, a cladding removed multimode optical fibre (CRMOF) and a single-mode multimode single-mode structure (SMS), are compared in terms of their adequateness for sensing once they are coated with thin-films. The thin-film deposited $\left(\mathrm{TiO}_{2} / \mathrm{PSS}\right)$ permits increasing the sensitivity to surrounding medium refractive index. The results obtained can be extrapolated to other fields such as biological or chemical sensing just by replacing the thin-film by a specific material.

\section{Introduction}

The deposition of thin-films on optical fibre substrates has permitted, during the last decade, widening the traditional domains of application of optical fibre sensors (e.g., strain and temperature [1]) to other research fields [2]. This is possible thanks to modern deposition techniques [3], which permit coating the optical fibre with materials, whose properties change as a function of a biological or chemical species. Moreover, the possibility of coating long period fibre gratings (LPFGs) with a material of thickness lower than one micrometer [4] has led to the observation of the mode transition phenomenon $[5,6]$, which has permitted developing optimized refractometers [6]. Moreover, even out of the mode transition region it is possible to obtain biosensors [7]. Recently, the combination of this phenomenon with the dispersion turning point [8] has permitted achieving sensitivities approaching $10000 \mathrm{~nm} / \mathrm{RIU}$ [9]. The mode transition consists of a reorganization of the effective indices of cladding modes when one of these modes is guided through the thin-film deposited on the cladding of an LPFG [5]. Recently, new simpler structures that do not require the inscription of a grating have been explored when a thin-film is deposited on the cladding. In this work two of them are compared: the cladding removed multimode optical fibre (CRMOF) and the single-mode multimode single-mode structure (SMS). Their properties are analyzed and their advantages and drawbacks are discussed.

\section{Experimental Setup}

In Figure 1 a transmission configuration setup is depicted for both the cladding removed multimode optical fibre (CRMOF) and the single-mode multimode single-mode structure (SMS). Light is launched into the optical fibre and the optical spectrum analyzer detects the light transmitted. In the sensitive region the CRMOF and SMS structures are coated with a thin-film.

A reflection setup is also presented in Figure 2 for the SMS structure. Light is launched into the optical fibre and reflected at the sensing head. The reflected light is detected at the optical spectrum analyzer. The sensing head is coated with a thin-film. The reason for not using the CRMOF with 


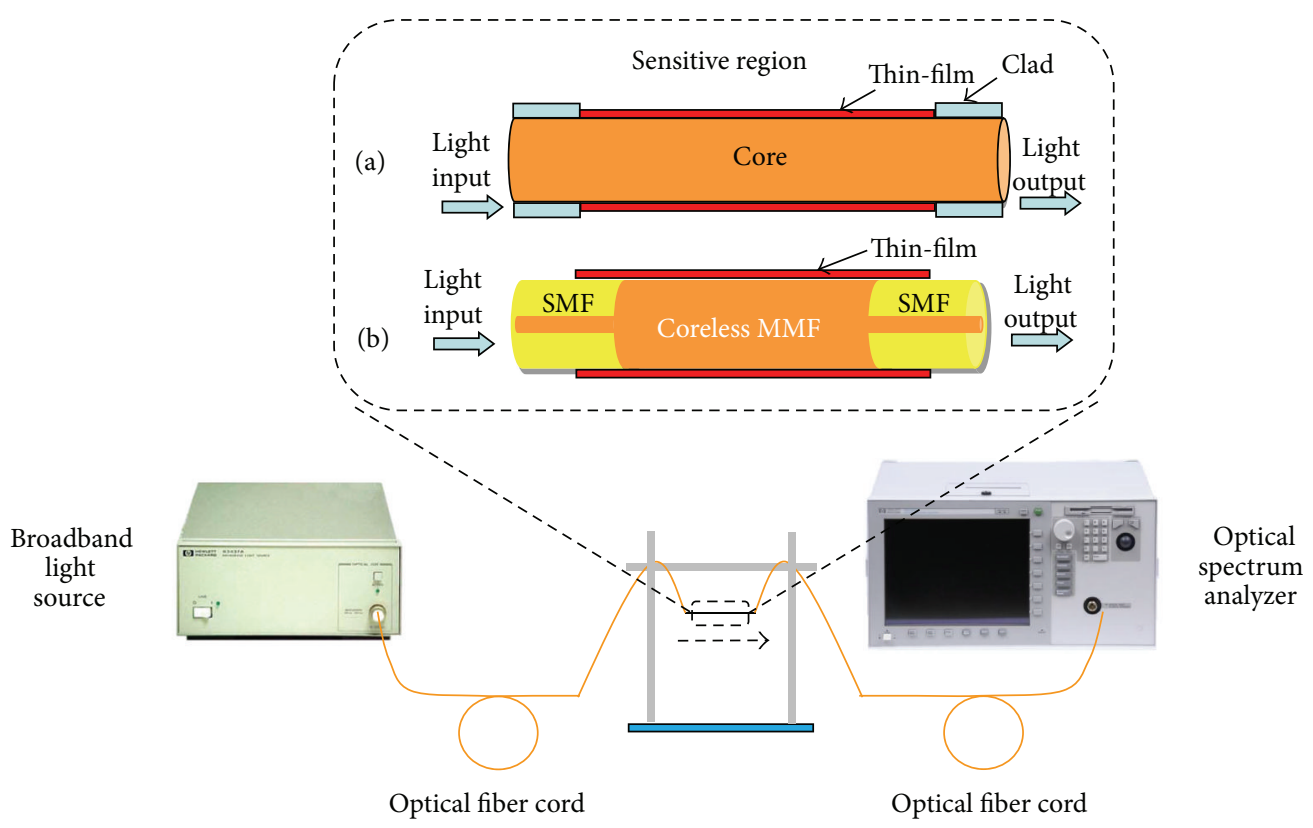

FIGURE 1: Transmission configuration setup for (a) cladding removed multimode optical fibre and (b) single-mode multimode single-mode structure.

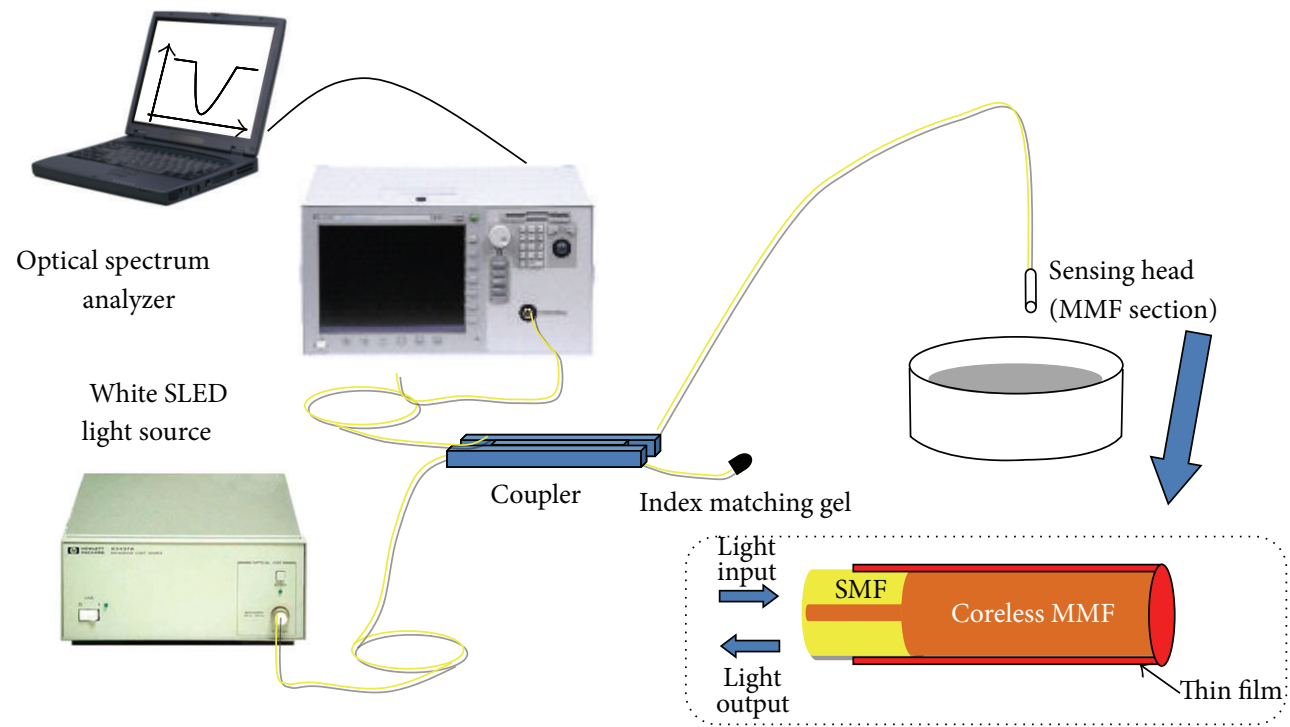

FIGURE 2: Reflection configuration setup for single-mode multimode single-mode structure.

the reflective configuration is that in some cases, as it will be shown later, the required thin-film thickness is higher, which leads to the influence of the interferometric phenomenon in the detection system.

The thin-film material in the experiments is $\mathrm{TiO}_{2} / \mathrm{PSS}[10$, 11], deposited with layer-by-layer self-assembly [12].

\section{Theory}

3.1. Cladding Removed Multimode Optical Fibre (CRMOF). For the simulation of the propagation of light through the the coated CRMOF, a method based on the attenuated total reflection (ATR) with a Kretschmann configuration is used [13]. The reflectivity as a function of wavelength and incidence angle is obtained at the coating-fibre core interface $R(\theta, \lambda)$ [14], where $\lambda$ is the light wavelength and $\theta$ the angle of incidence. The general expression for calculating the transmitted optical power is $[13,14]$

$$
T(\lambda)=\frac{\int_{\theta_{c}}^{90^{\circ}} p(\theta) R^{N(\theta)}(\theta, \lambda) d \theta}{\int_{\theta_{c}}^{90^{\circ}} p(\theta)},
$$


where $\theta_{c}$ is the critical angle, $N(\theta)$ is the number of reflections at the coating-fibre core interface, and $p(\theta)$ is the power distribution of the optical source. $R^{N(\theta)}(\theta, \lambda)$ represents the reflected light as a combination of the reflected power in TE and TM mode polarization [14].

3.2. Single-Mode-Multimode-Single-Mode Structure (SMS). For the calculation of light propagation in a SMS structure with transmission configuration (see Figure 1(a)), it is necessary to use a method based on the transfer matrix method [15], which consists of three steps [16]: calculation of the propagation constants of the mode in the single-mode fibre, the same for the modes in the coreless multimode segment, and derivation of the transmission and reflection coefficients.

The resonances in the transmission spectrum can be explained by a coupling from the core mode in the single mode segment to different modes in the MMF segment. The phase of modes at the end of the MMF segment is different and the coupling to another single mode section at the end of the MMF section causes constructive or destructive interference depending on the modal phase matching. The selfimaging effect permits obtaining the highest transmission in the optical spectrum and its central wavelength can be tuned by adequate selection of the parameters of the following expression [16]:

$$
Z_{i}=\frac{4 D^{2} n}{\lambda}
$$

where $D$ and $n$ are the MMF diameter and refractive index, respectively, and $\lambda$ is the operational wavelength.

For the SMS structure in reflection configuration (Figure 2), a slight modification of expression (2) permits obtaining the following:

$$
Z_{i}=\frac{2 D^{2} n}{\lambda}
$$

\section{Results and Discussion}

In Figure 3 the transmission through a $\mathrm{TiO}_{2} / \mathrm{PSS}$ coated CRMOF structure is plotted as a function of coating thickness and wavelength. Regions in blue color indicate a low transmission. This is explained in [13] as a lossy mode resonance phenomenon (LMR), which is produced when a particular mode guided in the optical fiber experiments a transition to guidance in a thin-film with a complex refractive index deposited on the optical fiber. Due to the complex refractive index of the thin-film, the mode guided in it owns also a complex refractive index. This specific mode is considered by some authors as a lossy mode $[17,18]$. Consequently, in $[10,11,13,19,20]$ the term lossy mode resonance is used instead of the term guided mode resonance [21]. Indeed, this lossy mode guided in the film is what induces the mode transition in LPFGs [19], a phenomenon that is also observed in the SMS structure [16].

In Figure 4 the central wavelength of each LMR is represented as a function of coating thickness and wavelength. Each squared marker in Figure 4 is the central wavelength

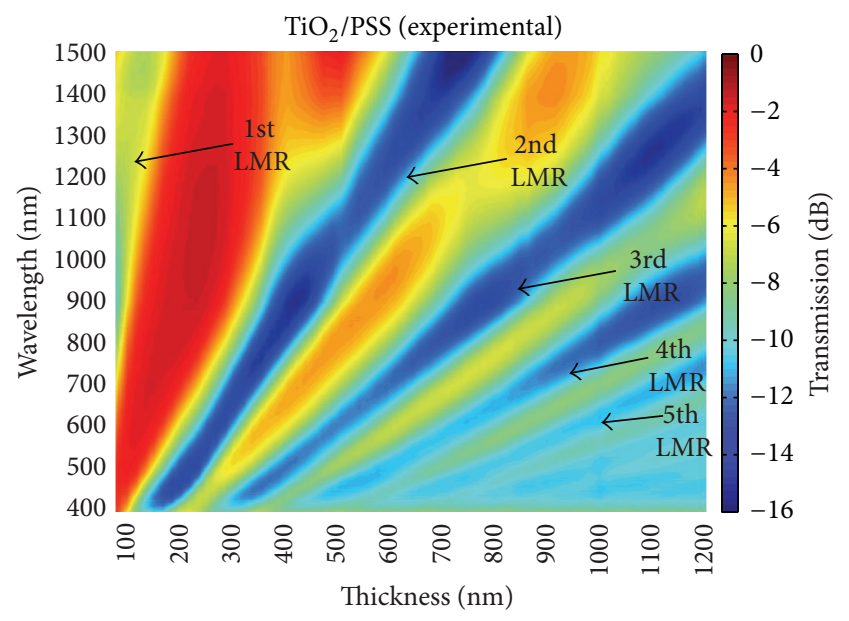

FIGURE 3: Spectral response obtained as a function of thickness for $\mathrm{TiO}_{2} /$ PSS coated CRMOF.

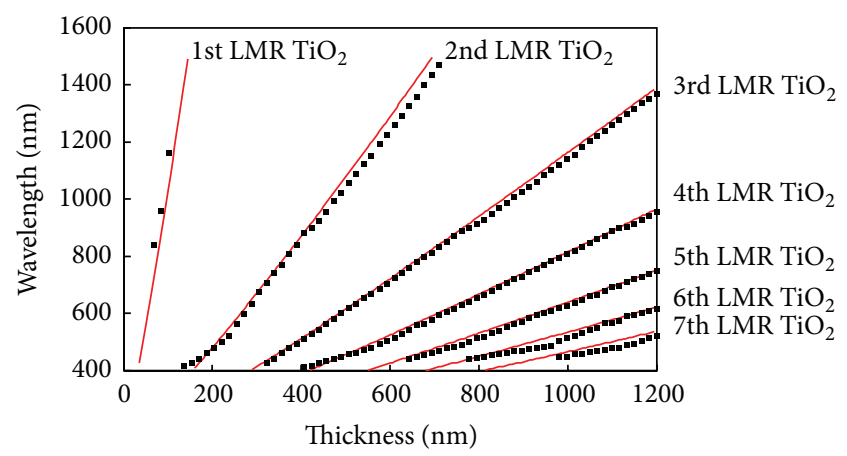

FIGURE 4: Evolution as a function of thickness of the central wavelength of LMRs for $\mathrm{TiO}_{2} /$ PSS coated CRMOF. Straight lines and squared markers corresponded, respectively, to theoretical and experimental results.

after the deposition of a bilayer and the straight lines are the theoretical results obtained with the numerical method of Section 2.

If single spectra for a specific thickness of 68 and $372 \mathrm{~nm}$ are plotted (see Figure 5), the bandwidth of the resonance depends on the thickness of the thin-film. If the rule considered for comparison of the bandwidth is the full width at half maximum (FWHM), it is not possible to obtain this parameter for a thin-film of $68 \mathrm{~nm}$ because the maximum value cannot be observed in the wavelength range analysed. In other words, the FWHM is higher than $1100 \mathrm{~nm}$ for the first LMR in Figure 3. However, for a thin-film of $372 \mathrm{~nm}$, the bandwidth for the FWHM centred at $800 \mathrm{~nm}$ is $366.8 \mathrm{~nm}$ (it belongs to the second LMR in Figure 3).

However, the reduction of the bandwidth leads also to a reduction of sensitivity. In Figure 6, plots of the wavelength shift as a function of the surrounding medium refractive index (SRI) for LMRs obtained with a $1165 \mathrm{~nm}$ coating (third, fourth, fifth, and sixth resonance) are presented. They show sensitivities of $1676,1018,611$, and $407 \mathrm{~nm} / \mathrm{RIU}$, respectively. 


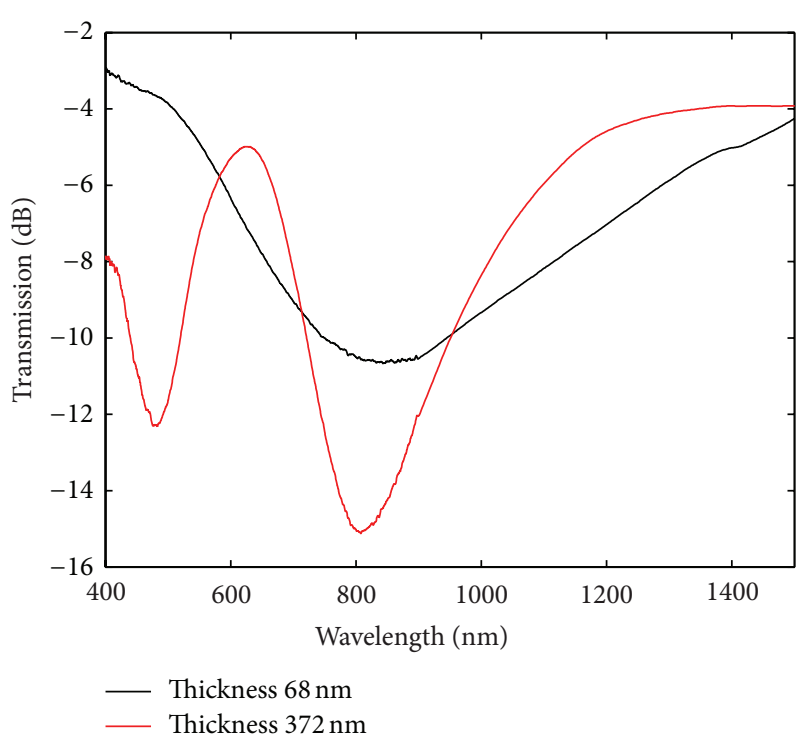

FIGURE 5: LMRs for CRMOF structure coated with 68 and $372 \mathrm{~nm}$ of $\mathrm{TiO}_{2} / \mathrm{PSS}$. With a thicker coating the LMR bandwidth is reduced (for $68 \mathrm{~nm}$ the first resonance is monitored and for $372 \mathrm{~nm}$ the second and the third resonance are visualized).

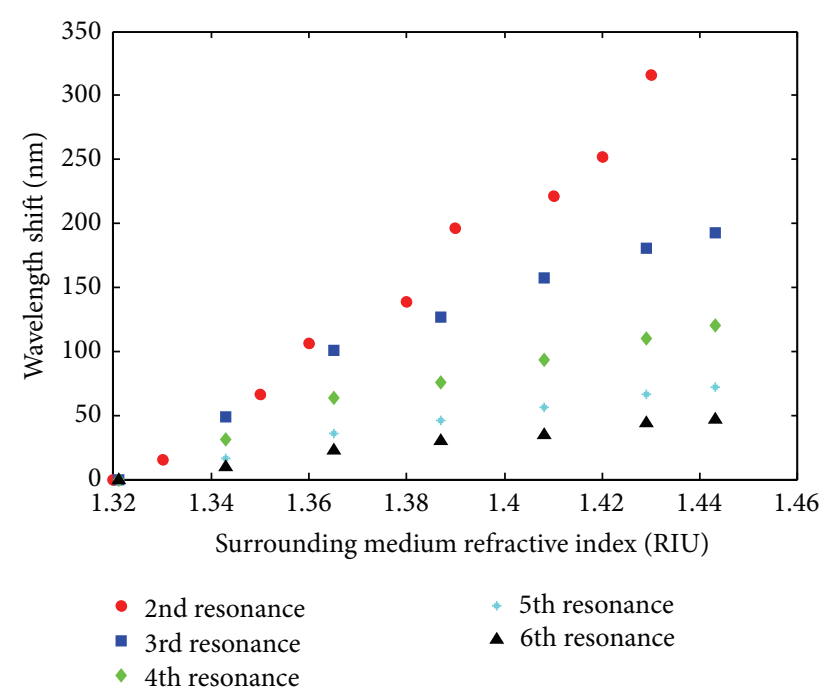

FIGURE 6: Central wavelength shift as a function of SRI for different LMRs obtained with a $\mathrm{TiO}_{2}$ /PSS coated CRMOF structure.

In addition to this, a plot for a $460 \mathrm{~nm}$ coating (second LMR) with a sensitivity of $2873 \mathrm{~nm} / \mathrm{RIU}$ is presented.

On the other hand, if the sensitivity to nanocoating thickness is analysed in Figure 4, the sensitivity of the second LMR is $2.04 \mathrm{~nm} / \mathrm{nm}$, whereas for the first one it is $9.66 \mathrm{~nm} / \mathrm{nm}$, which indicates almost a 5-fold increase. If this improvement is extrapolated for calculation of the sensitivity to refractive index of the first LMR, the record sensitivity to SRI in the range 1.345-1.36 obtained in [9] with LPFGs (5924 nm/RIU) could be overcome. However, coated CRMOFs present a problem of a high bandwidth.

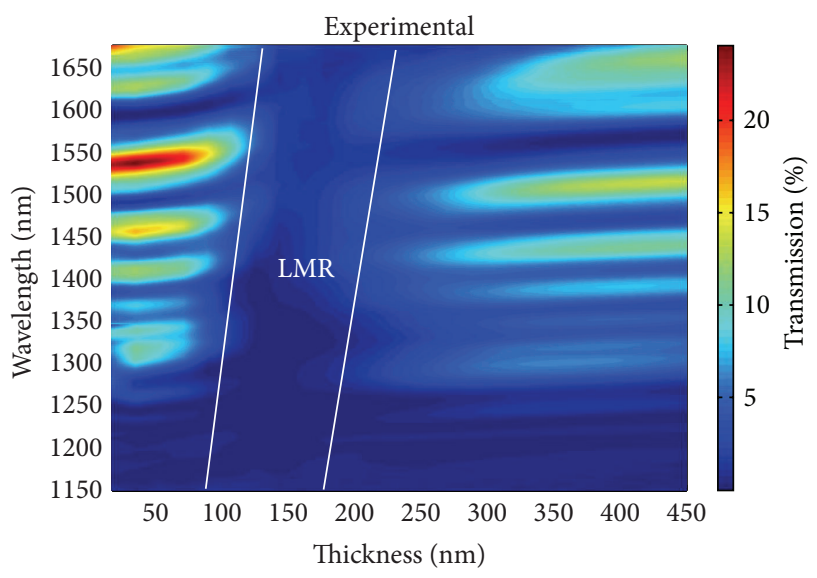

FIGURE 7: Evolution as a function of thickness of the optical spectrum for a SMS structure coated with $\mathrm{TiO}_{2} / \mathrm{PSS}$ with section length and diameter of $20 \mathrm{~mm}$ and $125 \mu \mathrm{m}$, respectively.

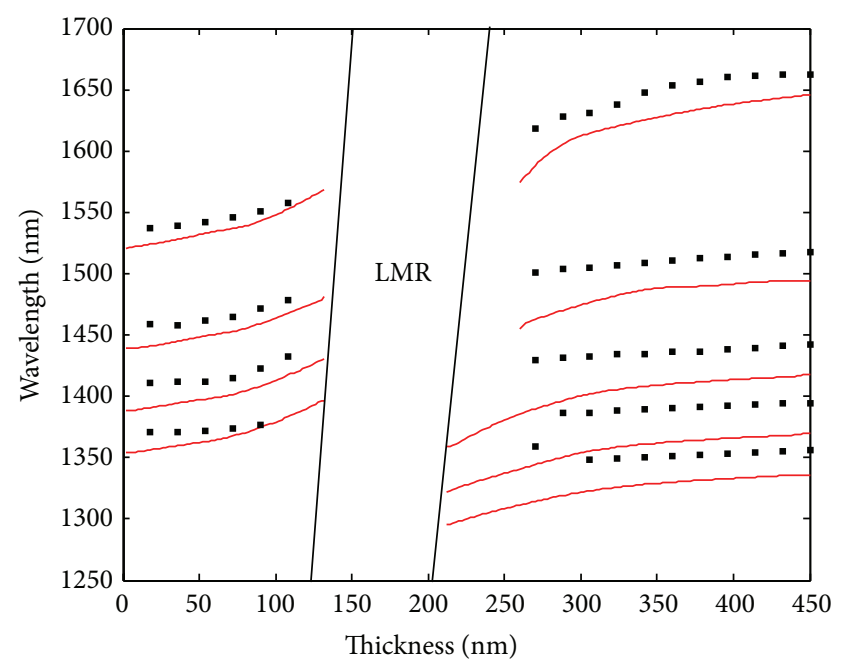

FIGURE 8: Evolution as a function of thickness of the central wavelength of transmission bands obtained with an SMS structure with MMF section length and diameter, respectively, $20 \mathrm{~mm}$ and $125 \mu \mathrm{m}$. Straight lines and squared markers corresponded, respectively, to theoretical and experimental results.

If the same thin-film material is deposited on an SMS structure, a plot that resembles that of LPFGs [4-6] is obtained (see Figure 7). Initially the transmission bands (the regions in red colour) present a wavelength shift until a fading region is reached. As it was proved with LPFGs, this band coincides with the LMR region [19]. After this region, the bands are again visible.

In Figure 8 the central wavelength of the bands is tracked as a function of the number of thin-film thickness. Each squared marker in Figure 8 is the central wavelength of the transmission band after the deposition of a bilayer and the straight lines are the theoretical results obtained with the numerical method of Section 2.

In Figure 9 the evolution of the optical spectrum as a function of the number of bilayers of $\mathrm{TiO}_{2} / \mathrm{PSS}$ is represented 


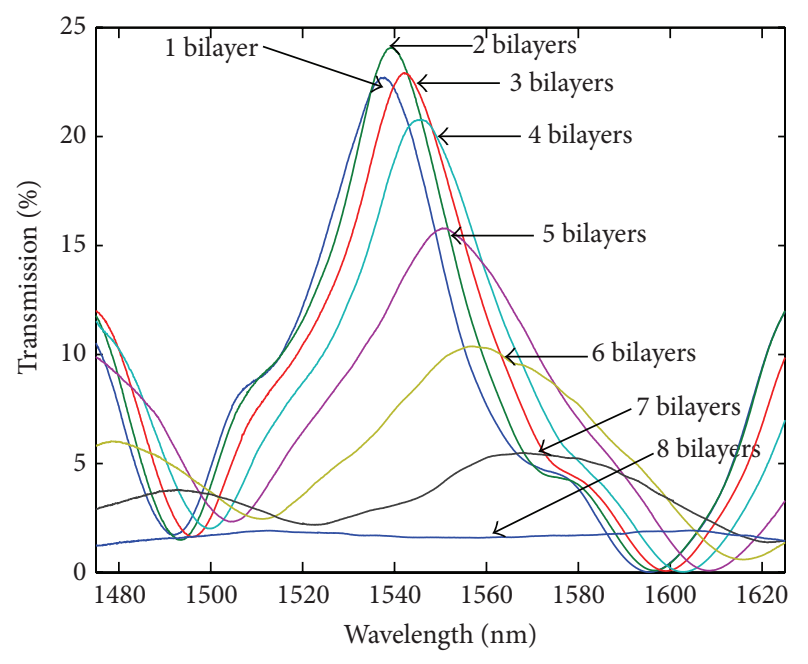

FIGURE 9: Evolution as a function of thickness of one of the transmission bands obtained with an SMS structure with MMF section length and diameter of $20 \mathrm{~mm}$ and $125 \mu \mathrm{m}$, respectively.

(the estimated bilayer thickness $18 \mathrm{~nm}$ ). The resonance progressively vanishes for 6 bilayers (108 $\mathrm{nm}$ ) or more.

In view that the results were obtained in water $(S R I=$ 1.33), if a refractometer is to be developed for refractive indices not exceeding that of water (1.33), it is a good design to stop at 6 bilayers. However, if a refractometer for higher refractive indices is to be developed, then one must be more cautious and stop for a lower number of bilayers.

In Figure 10 the wavelength shifts for different thickness values are analyzed theoretically. As the thickness increases, the sensitivity is improved, but the range of refractive indices that can be analyzed without entering the fading region is reduced. In addition to this, in all cases the sensitivity improves for the SMS structure with a lower diameter in the MMF section.

In order to obtain an easy to handle device, an SMS in reflective configuration is designed (see Figure 2). The deposition of the thin-film also at the end of the structure should induce an interferometer [22]. However, the thickness of the thin-film is low enough to guarantee that interferometric effects do not play a significant role in the results [23]. This is an advantage in comparison with CRMOF structures that, depending on the resonance that is being tracked, require a higher or lower thin-film thickness. If the thin-film thickness is high, then interferometry should disturb the visibility of the LMR. In this case, the solution is to set a mirror at the end of the optical fibre structure [24], something that is not necessary with the SMS structure.

In Figure 11, the experimental self-image band can be observed for both SMS structures analysed theoretically in Figure 10. The FWHM is $16.7 \mathrm{~nm}$ for the SMS structure with MMF section length of $125 \mu \mathrm{m}$ and $28.9 \mathrm{~nm}$ for the SMS structure with MMF section length $61.5 \mu \mathrm{m}$. The thin-film deposited on both structures consisted of the deposition of $5 \mathrm{TiO}_{2} / \mathrm{PSS}$ bilayers with an estimated thickness per bilayer of $17 \mathrm{~nm}$. This number of bilayers permits the transmission
TABLE 1: FWHM, sensitivity to SRI and figure of merit of CRMOF, SMS with diameter $125 \mu \mathrm{m}$, and SMS with diameter $61.5 \mu \mathrm{m}$.

\begin{tabular}{lccc}
\hline & $\begin{array}{c}\text { Sensitivity } \\
(\mathrm{nm} / \mathrm{RIU})\end{array}$ & $\begin{array}{c}\text { FWHM } \\
(\mathrm{nm})\end{array}$ & $\begin{array}{c}\text { Sensitivity/FWHM } \\
\left(\mathrm{RIU}^{-1}\right)\end{array}$ \\
\hline CRMOF & 2873 & 366.8 & 7.8 \\
$\begin{array}{l}\text { SMS (diameter } \\
125 \mu \mathrm{m})\end{array}$ & 631.2 & 16.7 & 37.8 \\
$\begin{array}{l}\text { SMS (diameter } \\
61.5 \mu \mathrm{m})\end{array}$ & 1199.2 & 28.9 & 41.5 \\
\hline
\end{tabular}

band to approach the vanishing region for the SRI range analysed in Figure 12, where the sensitivities are, respectively, 631 and $1199 \mathrm{~nm} / \mathrm{RIU}$. These values are, respectively, 4 and 2 times lower than that obtained with the thin-film coated CRMOF $(2873 \mathrm{~nm} / \mathrm{RIU})$. However, the FWHM is 10 times and 5 times lower than the FWHM of the thin-film coated CRMOF structure.

If a figure of merit based on the ratio between the sensitivity to SRI and the FWHM is used [25], values of 37.78, 41.49, and 7.83 are obtained, respectively, for an SMS structure with MMF section diameter $125 \mu \mathrm{m}$, for an SMS structure with MMF section diameter $61.5 \mu \mathrm{m}$, and for the CRMOF, respectively (see Table 1). This indicates that the figure of merit of SMS structures is five-fold that of CRMOF.

\section{Conclusions}

It has been successfully proved that optical fibre structures without the inscription of a grating can be used for wavelength based sensors if a nanocoating is deposited on the cladding. The different properties of the two structures studied, cladding removed multimode optical fibre (CRMOF) and single-mode multimode single-mode structure (SMS), permit choosing the most adequate device depending on the needs of the final user. If sensitivity is the most important thing, CRMOF devices are the best option (they can even overcome the sensitivity obtained with LPFGs). However, if the figure of merit, considered as the ratio between the sensitivity and the bandwidth of the resonance is considered, the best option is the SMS structure. In addition to this, it has been proved that a simple and reflective configuration without the need of a mirror is possible with the SMS structure, something that is not so easy to control with a CRMOF (this is only possible with a low thin-film thickness or with a mirror at the optical fibre tip).

The results obtained have been focused on the detection of the refractive index. However, by choosing a material for the thin-film that is sensitive to a specific species, chemical or biological sensors can be developed.

\section{Conflict of Interests}

The authors declare that there is no conflict of interests regarding the publication of this paper. 


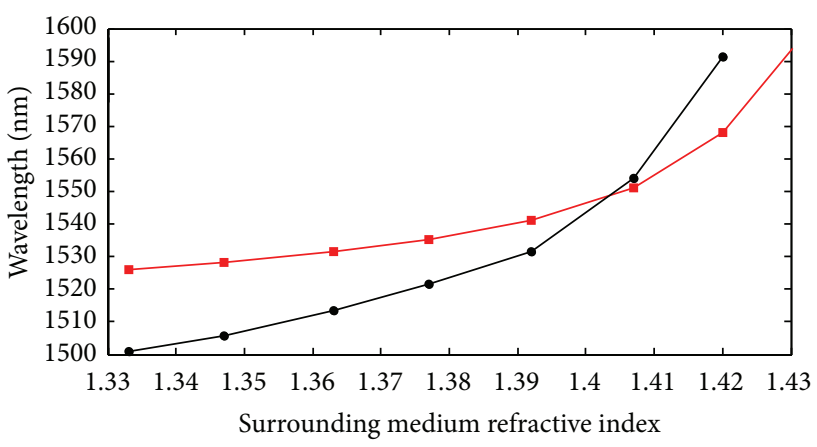

- SMS 125 micron $\left(\mathrm{TiO}_{2}\right.$ coating) thickness: $26 \mathrm{~nm}$

$\rightarrow$ SMS 61.5 micron $\left(\mathrm{TiO}_{2}\right.$ coating) thickness: $26 \mathrm{~nm}$

(a)

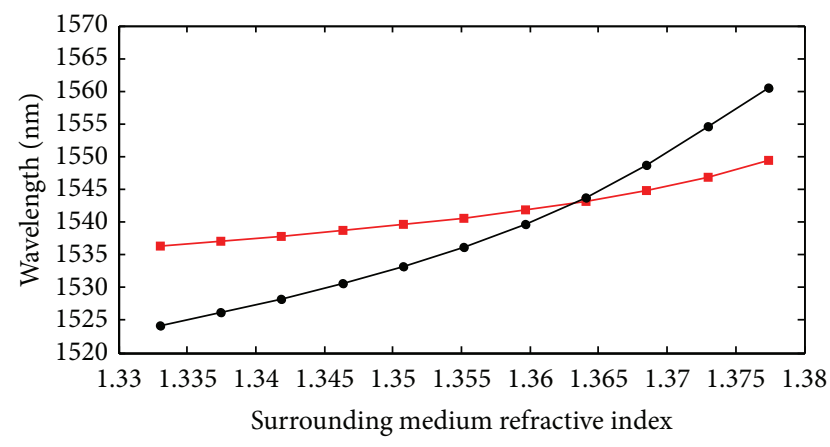

- SMS 125 micron ( $\mathrm{TiO}_{2}$ coating) thickness: $65 \mathrm{~nm}$

$\rightarrow$ SMS 61.5 micron $\left(\mathrm{TiO}_{2}\right.$ coating) thickness: $65 \mathrm{~nm}$

(b)

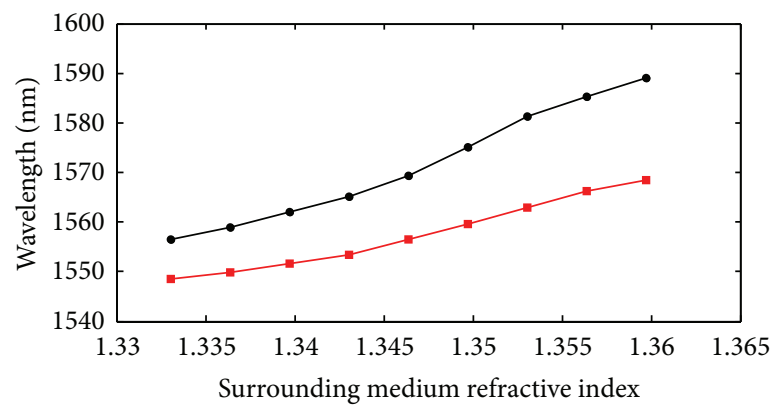

$\rightarrow$ SMS 125 micron ( $\mathrm{TiO}_{2}$ coating) thickness: $104 \mathrm{~nm}$

$\rightarrow$ SMS 61.5 micron $\left(\mathrm{TiO}_{2}\right.$ coating) thickness: $104 \mathrm{~nm}$

(c)

FIGURE 10: Numerical results: central wavelength of transmission bands obtained with a SMS structure with MMF section length 20 mm and diameters 125 and $61.5 \mu \mathrm{m}$ as a function of the SRI. Coating thickness: (a) $26 \mathrm{~nm}$, (b) $65 \mathrm{~nm}$, and (c) $104 \mathrm{~nm}$.

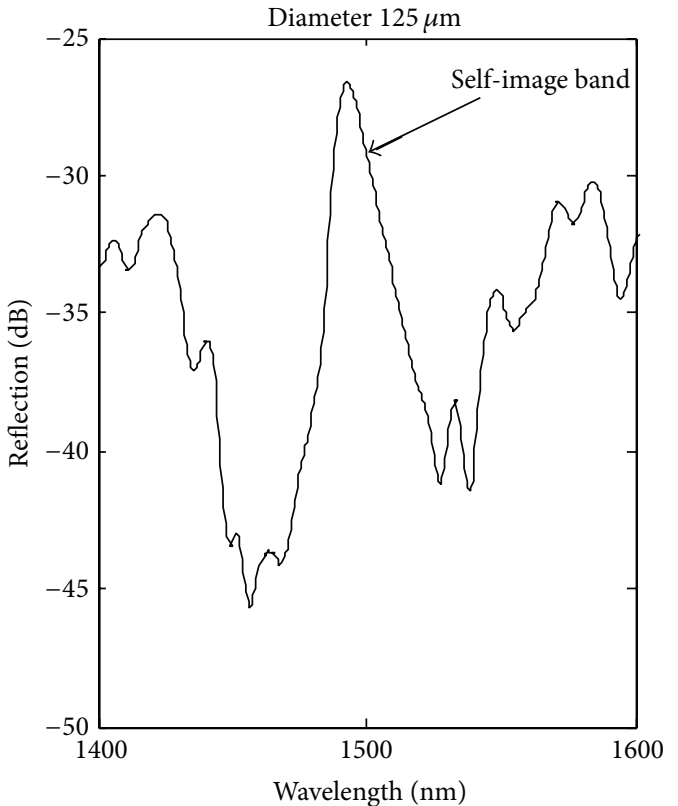

(a)

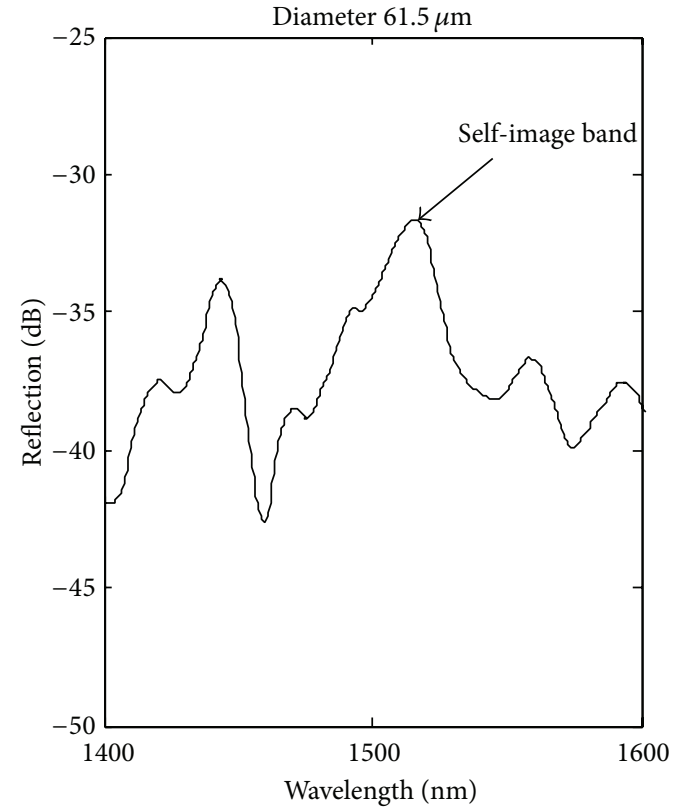

(b)

FIGURE 11: Self-image band for an SMS structure with an MMF segment of diameter: (a) $125 \mu \mathrm{m}$ and (b) $61.5 \mu \mathrm{m}$. 


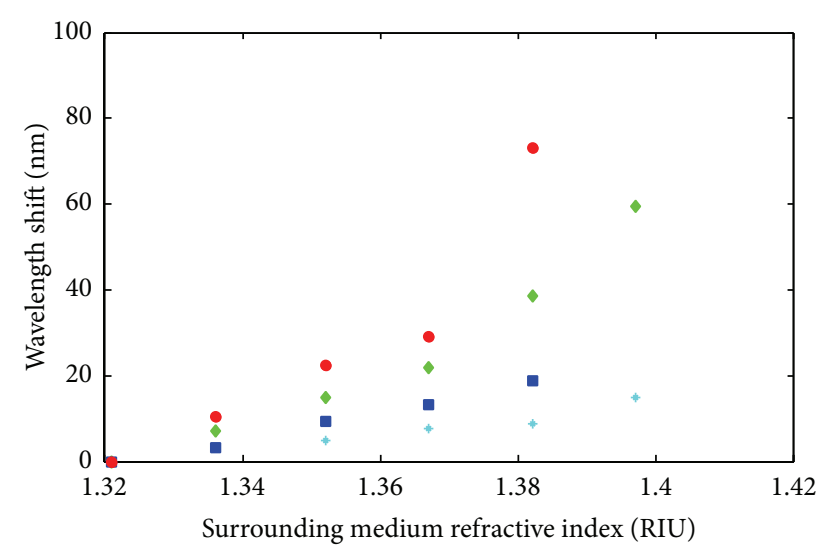

* Without deposition diameter $125 \mu \mathrm{m}$

- With 5 bilayers diameter $125 \mu \mathrm{m}$

- Without deposition diameter $61.5 \mu \mathrm{m}$

- With 5 bilayers diameter $61.5 \mu \mathrm{m}$

FIGURE 12: Central wavelength shift as a function of SRI for an SMS structure with and without deposition of a thin film for MMF segment of diameters $125 \mu \mathrm{m}$ and $61.5 \mu \mathrm{m}$.

\section{Acknowledgment}

This work was supported in part by the Spanish Ministry of Education and Science-FEDER TEC2013-43679-R.

\section{References}

[1] B. Lee, "Review of the present status of optical fiber sensors," Optical Fiber Technology, vol. 9, no. 2, pp. 57-79, 2003.

[2] C. R. Zamarreño, J. M. Corres, J. Goicoechea, I. Del Villar, I. R. Matias, and F. J. Arregui, "Fibre optic nanosensors," in Optochemical Nanosensors, A. Cusano, F. J. Arregui, M. Giordano, and A. Cutolo, Eds., pp. 167-203, Taylor \& Francis, New York, NY, USA, 2012.

[3] H. S. Nalwa, Handbook of Thin Films, Volume 1: Deposition and Processing of Thin Films, Elsevier, 2002.

[4] N. D. Rees, S. W. James, R. P. Tatam, and G. J. Ashwell, "Optical fiber long-period gratings with Langmuir-Blodgett thin-film overlays," Optics Letters, vol. 27, no. 9, pp. 686-688, 2002.

[5] I. D. Villar, I. R. Matías, F. J. Arregui, and P. Lalanne, “Optimization of sensitivity in Long Period Fiber Gratings with overlay deposition," Optics Express, vol. 13, no. 1, pp. 56-69, 2005.

[6] A. Cusano, A. Iadicicco, P. Pilla et al., "Mode transition in high refractive index coated long period gratings," Optics Express, vol. 14, no. 1, pp. 19-34, 2006.

[7] E. Brzozowska, M. Śmietana, M. Koba et al., "Recognition of bacterial lipopolysaccharide using bacteriophage-adhesincoated long-period gratings," Biosensors and Bioelectronics, vol. 67, pp. 93-99, 2015.

[8] C. S. Cheung, S. M. Topliss, S. W. James, and R. P. Tatam, "Response of fiber-optic long-period gratings operating near the phase-matching turning point to the deposition of nanostructured coatings," Journal of the Optical Society of America B, vol. 25, no. 6, pp. 897-902, 2008.

[9] P. Pilla, C. Trono, F. Baldini, F. Chiavaioli, M. Giordano, and A. Cusano, "Giant sensitivity of long period gratings in transition mode near the dispersion turning point: an integrated design approach," Optics Letters, vol. 37, no. 19, pp. 4152-4154, 2012.

[10] M. Hernáez, I. Del Villar, C. R. Zamarreño, F. J. Arregui, and I. R. Matias, "Optical fiber refractometers based on lossy mode resonances supported by $\mathrm{TiO}_{2}$ coatings," Applied Optics, vol. 49, no. 20, pp. 3980-3985, 2010.

[11] N. Paliwal and J. John, "Theoretical modeling of lossy mode resonance based refractive index sensors with $\mathrm{ITO} / \mathrm{TiO}_{2}$ bilayers," Applied Optics, vol. 53, no. 15, pp. 3241-3246, 2014.

[12] G. Decher, "Fuzzy nanoassemblies: toward layered polymeric multicomposites," Science, vol. 277, no. 5330, pp. 1232-1237, 1997.

[13] I. Del Villar, C. R. Zamarreño, M. Hernaez, F. J. Arregui, and I. R. Matias, "Lossy mode resonance generation with indium-tinoxide-coated optical fibers for sensing applications," Journal of Lightwave Technology, vol. 28, no. 1, pp. 111-117, 2010.

[14] Y. Xu, N. B. Jones, J. C. Fothergill, and C. D. Hanning, "Analytical estimates of the characteristics of surface plasmon resonance fibre-optic sensors," Journal of Modern Optics, vol. 47, no. 6, pp. 1099-1110, 2000.

[15] G. W. Chern and L. A. Wang, "Transfer-matrix method based on perturbation expansion for periodic and quasi-periodic binary long-period gratings," Journal of the Optical Society of America A: Optics and Image Science, and Vision, vol. 16, no. 11, pp. 2675-2689, 1999.

[16] A. B. Socorro, I. Del Villar, J. M. Corres, F. J. Arregui, and I. R. Matias, "Mode transition in complex refractive index coated single-mode-multimode-single-mode structure," Optics Express, vol. 21, no. 10, pp. 12668-12682, 2013.

[17] T. E. Batchman and G. M. McWright, "Mode coupling between dielectric and semiconductor planar waveguides," IEEE Journal of Quantum Electronics, vol. 18, no. 4, pp. 782-788, 1982.

[18] M. Marciniak, J. Grzegorzewski, and M. Szustakowski, "Analysis of lossy mode cut-off conditions in planar waveguides with semiconductor guiding layer," IEE proceedings J. Optoelectronics, vol. 140, no. 4, pp. 247-252, 1993.

[19] I. Del Villar, C. R. Zamarreño, M. Hernáez, F. J. Arregui, and I. R. Matías, "Resonances in coated long period fiber gratings and cladding removed multimode optical fibers: a comparative study," Optics Express, vol. 18, no. 19, pp. 20183-20189, 2010.

[20] D. Kaur, V. K. Sharma, and A. Kapoor, "High sensitivity lossy mode resonance sensors," Sensors and Actuators, B: Chemical, vol. 198, pp. 366-376, 2014.

[21] F. Yang and J. R. Sambles, "Determination of the optical permittivity and thickness of absorbing films using long range modes," Journal of Modern Optics, vol. 44, no. 6, pp. 1155-1163, 1997.

[22] F. J. Arregui, I. R. Matias, Y. Liu, K. M. Lenahan, and R. O. Claus, "Optical fiber nanometer-scale Fabry-Perot interferometer formed by the ionic self-assembly monolayer process," Optics Letters, vol. 24, no. 9, pp. 596-598, 1999.

[23] I. Del Villar, A. B. Socorro, J. M. Corres, F. J. Arregui, and I. R. Matias, "Refractometric sensors based on multimode interference in a thin-film coated single-mode-multimode-singlemode structure with reflection configuration," Applied Optics, vol. 53, no. 18, pp. 3913-3919, 2014.

[24] D. W. Kim, Y. Zhang, K. L. Cooper, and A. Wang, "Fibre-optic interferometric immuno-sensor using long period grating," Electronics Letters, vol. 42, no. 6, pp. 324-325, 2006.

[25] B. Špačková and J. Homola, "Sensing properties of lattice resonances of 2D metal nanoparticle arrays: an analytical model," Optics Express, vol. 21, no. 22, pp. 27490-27502, 2013. 

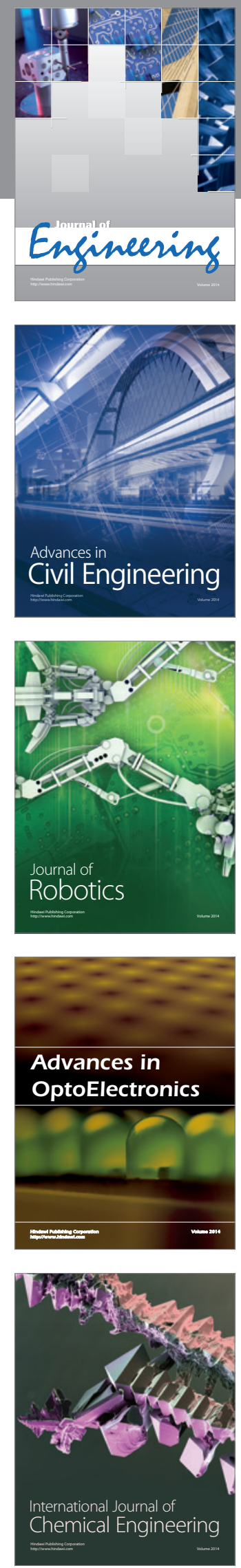

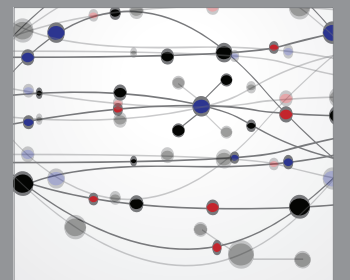

The Scientific World Journal
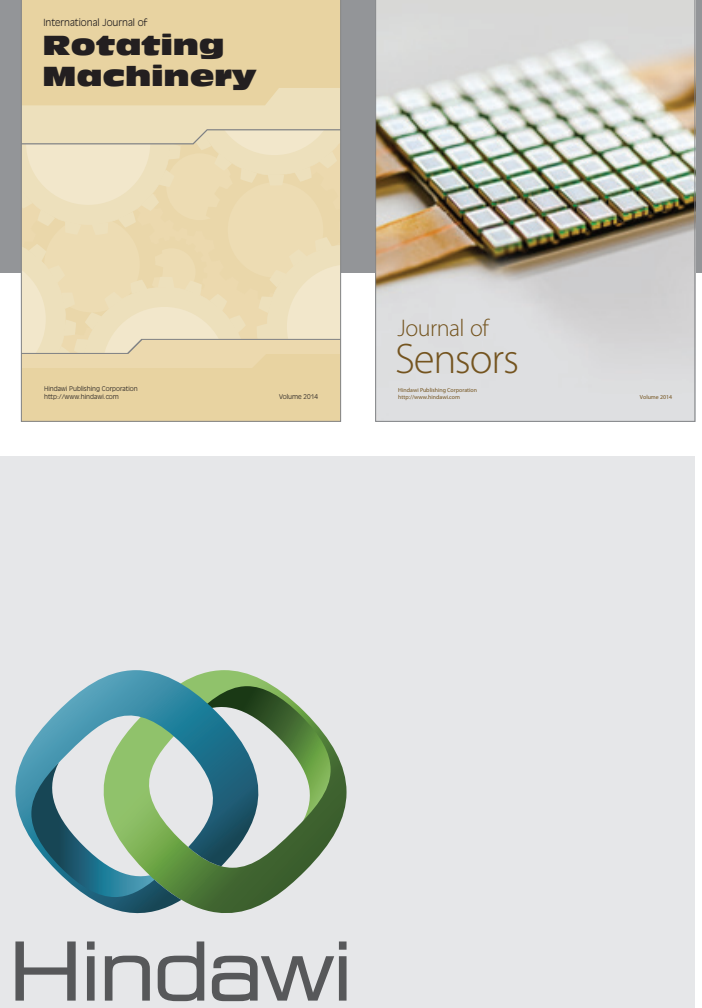

Submit your manuscripts at http://www.hindawi.com
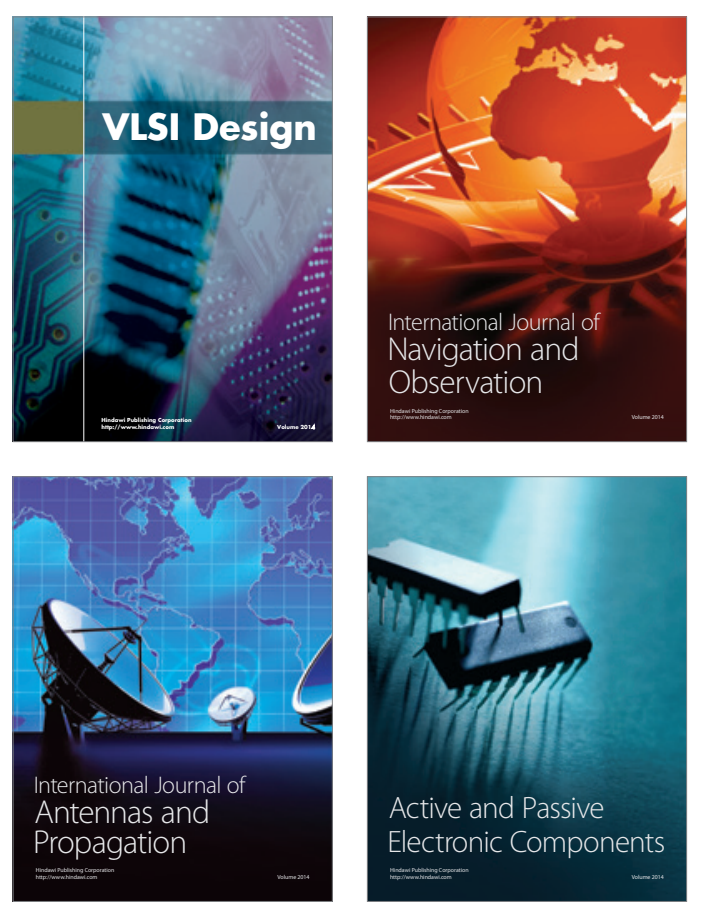
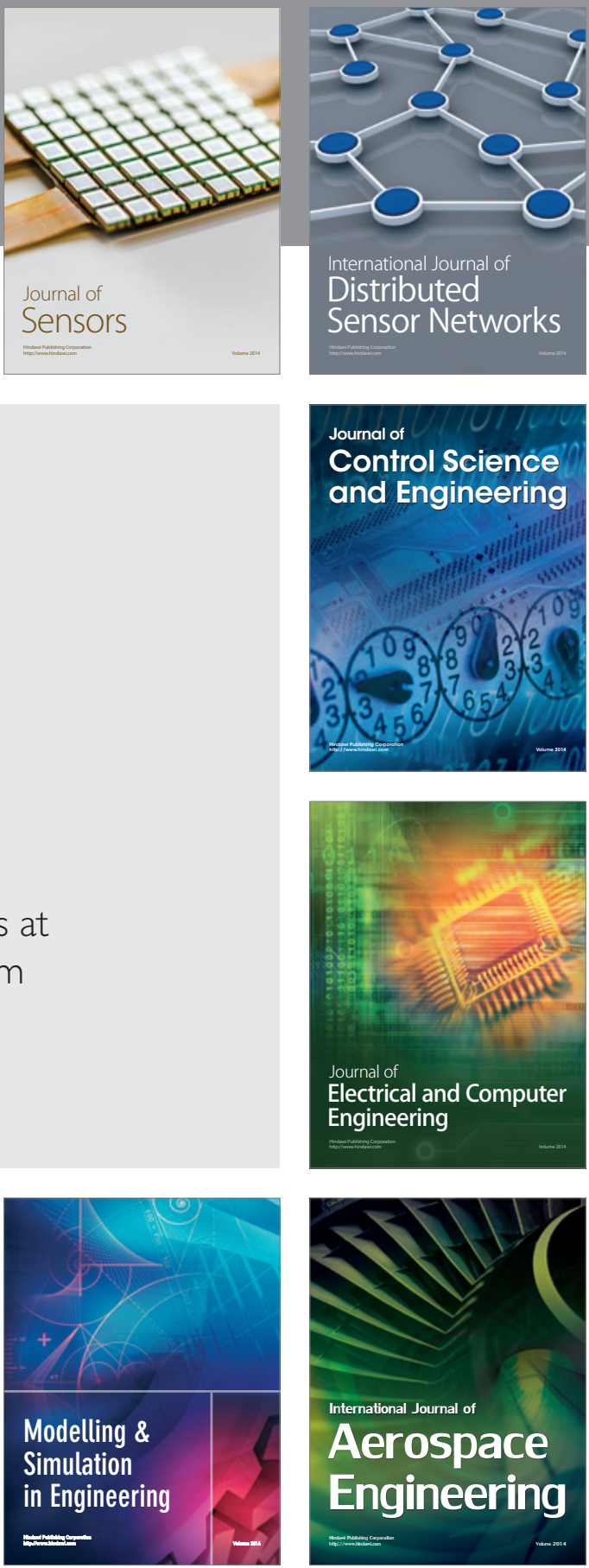

Journal of

Control Science

and Engineering
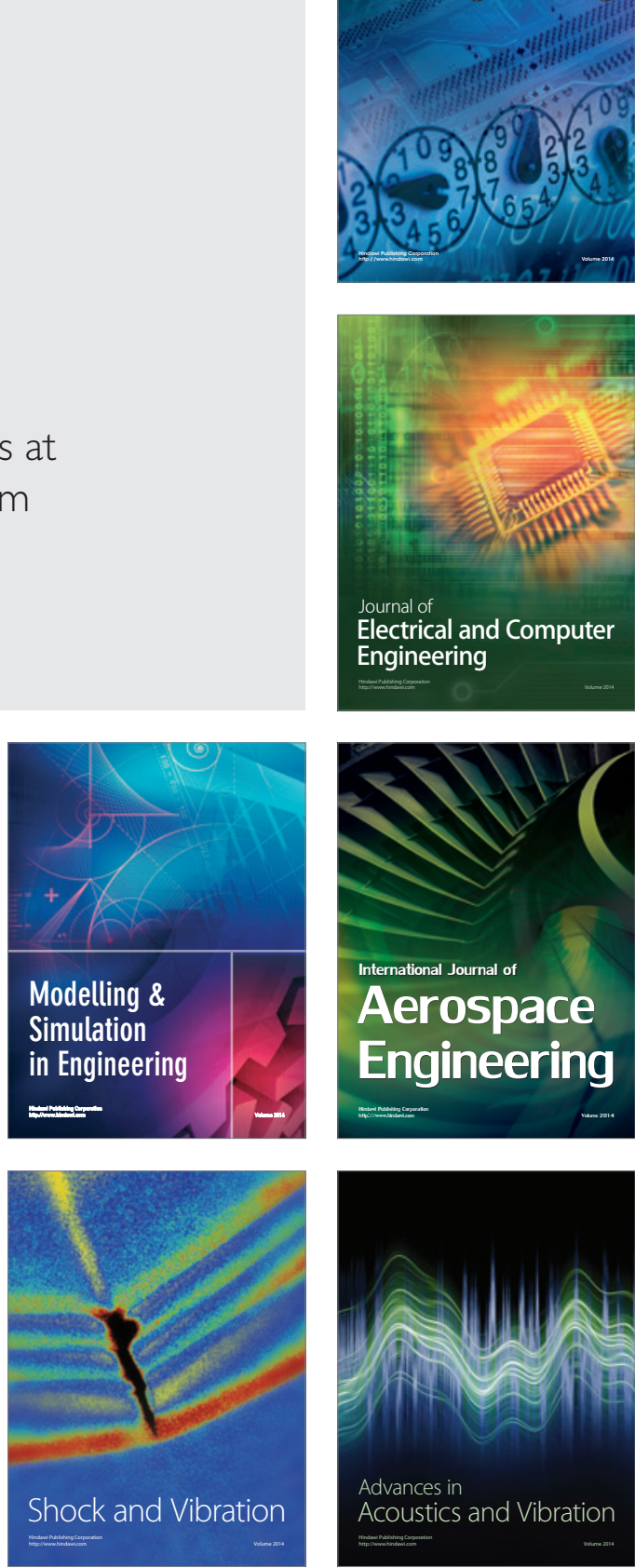